\title{
MALNUTRICIÓN EN PACIENTES CON PATOLOGÍA QUIRÚRGICA EN CIRUGÍA GENERAL
}

\section{Hospital de San José, marzo 2009 a agosto 2009}

\author{
Juan Pablo Ruiz P. MD*, Óscar García MD**, Claudia Medina***
}

\section{Resumen}

Introducción: la malnutrición es un proceso mórbido serio con múltiples causas de índole clínica y socioeconómica. Tiene desenlaces desde el punto de vista de morbimortalidad, estancia hospitalaria prolongada así como de costos de atención en salud. Existen múltiples métodos directos e indirectos que pueden ser útiles en la detección temprana. La valoración global subjetiva (VGS) es uno simple y fácil de aplicar a los pacientes hospitalizados y es válida en la práctica clínica. Objetivo: determinar la frecuencia de malnutrición de los pacientes con patología quirúrgica hospitalizados en el servicio de cirugía general del Hospital de San José a través de la VGS. Métodos: se realizó un estudio descriptivo. Los criterios de inclusión fueron pacientes hospitalizados mayores de 18 años con una estancia mínima de 24 horas en el servicio de cirugía general, que no tuvieran asistencia del grupo de soporte nutricional, a los cuales se les aplicó la VGS. El análisis se realizó en STATA 10. Resultados: se estudiaron 281 casos. La frecuencia de malnutrición fue de $45,2 \%$, siendo el $12,8 \%$ severa. La mediana de la edad fue 45 años. El $70 \%$ de los desnutridos tuvieron cirugía abdominal. La estancia fue más prolongada en los pacientes malnutridos. Conclusión: en este estudio se analizaron 281 pacientes durante seis meses, con una prevalencia de malnutrición del $\mathbf{4 5 , 2} \%$, que es alta, pero se encuentra cerca de lo reportado en la literatura médica latinoamericana. La mayoría de los pacientes malnutridos fueron los posquirúrgicos dado por el aumento de su catabolismo.

Palabras clave: malnutrición hospitalaria, valoración global subjetiva, soporte nutricional, desnutrición.

Abreviaturas: VGS, valoración global subjetiva; MH, malnutrición hospitalaria.

\section{Abstract}

Introduction: malnutrition is a serious morbid process caused by multiple clinical, social/economic factors. From the morbidity/ mortality point of view, it contributes to clinical outcomes, a longer hospital stay, as well as to health care costs. There are many direct and indirect useful methods for early detection. The Subjective Global Assessment (SGA) is a simple and easy method to apply on hospitalized patients and is valid in clinical practice. Objective: to determine the frequency of malnutrition on surgical patients hospitalized at the General Surgery Department at Hospital de San José using the SGA method. Methods: a descriptive study was performed. Inclusion criteria were: hospitalized patients, $=18$ years old, with at least a 24-hour hospital stay at the General Surgery Department, who did not receive assistance by the nutrition support group, who completed the SGA method survey. Data analysis was made by STATA 10. Results: 281 cases were studied. The frequency of malnutrition was $45.2 \%$, and $12.8 \%$ were rated as severe. The mean age was 45 years. Seventy percent $(70 \%)$ of malnourished patients had abdominal surgery. Hospital stay was longer in malnourished patients. Conclusion: 281 patients were analyzed during six months. The prevalence of malnutrition was $\mathbf{4 5 . 2 \%}$, which is high. However, this result is close to that reported in Latin American medical literature. Malnourishment resulting from increased catabolism was found mostly in post-operative patients.

Key Words: hospital malnutrition, Subjective Global Assessment, nutrition support, malnutrition.

Fecha recibido: marzo 8 de 2010 - Fecha aceptado: abril 29 de 2010

* Residente IV Cirugía General, Fundación Universitaria de Ciencias de la Salud, Hospital de San José, Bogotá DC. Colombia.

** Instructor Asociado, Cirugía General, Fundación Universitaria de Ciencias de la Salud. Cirujano de Seno y Tejidos Blandos, Hospital de San José, Bogotá DC. Colombia.
*** Nutricionista-dietista, Jefe del Servicio de Nutrición, Hospital de San José. Docente Fundación Universitaria de Ciencias de la Salud, Bogotá DC. Colombia.

Asesora metodológica: Merideidy Plazas. Docente División de Investigaciones. Fundación Universitaria de Ciencias de la Salud. Bogotá DC. Colombia. 


\section{Introducción}

La malnutrición hospitalaria es causa frecuente de mortalidad y uno de los principales problemas de salud en todo el mundo. Afecta en forma especial a un colectivo concreto como es el de los sujetos hospitalizados, en quienes la incapacidad, la enfermedad y las complicaciones son comunes, tomando nominación propia bajo el concepto de malnutrición hospitalaria $(\mathrm{MH}) .{ }^{1,2}$ El objetivo de la valoración es diagnosticar el estado nutricional (estado nutricional deficiente) y crear opciones de tratamiento para disminuir la morbilidad y la mortalidad. ${ }^{3}$ La prevalencia de MH reportada en la literatura es alta. En un estudio multicéntrico realizado en Latinoamérica con la participación de trece países (Argentina, Brasil, Chile, Costa Rica, Cuba, República Dominicana, México, Panamá, Paraguay, Perú, Puerto Rico, Venezuela y Uruguay) aplicando a pacientes hospitalizados la VGS, dio como resultado una prevalencia de malnutrición de $50,2 \% .^{2}$

El estudio realizado por Baccaro y cols. en Argentina ${ }^{1}$ utilizando la VGS encontró una prevalencia de $\mathrm{MH}$ de $48 \%$, que se asemeja con la reportada en la literatura médica. Lo anterior soporta la necesidad de implementar la VGS como requisito en la admisión hospitalaria de los pacientes para poder detectar la malnutrición y así poder disminuir las tasas altas de MH. La malnutrición ejerce un impacto negativo sobre la tasa de cicatrización y el funcionamiento no sólo del sistema inmunológico sino también de los diferentes órganos de la economía. ${ }^{4,5}$ Esto se refleja en que los pacientes desnutridos tienen veinte veces más posibilidades de complicaciones como aumento en las tasas de infección, hospitalización prolongada y alteración en el proceso de cicatrización de los tejidos, entre otras. ${ }^{4,6}$

Existen diversas técnicas para realizar la valoración nutricional sin que haya alguna considerada como estándar ideal, por tanto, la técnica adecuada será aquella que resulte fácil de aplicar, universal, precoz, basada en la mejor evidencia científica disponible y adaptable a las diversas circunstancias clínicas de los pacientes como edad, sexo y gravedad de la enfermedad. ${ }^{3,7}$ Dentro de las múltiples existentes en la actualidad, la VGS descrita por Detsky en 1987, es un instrumento clínico de evalua- ción del estado nutricional fácil de utilizar, de corta duración y que presenta una buena correlación de los resultados entre examinadores cuando ellos están bien entrenados. ${ }^{3,7}$

\section{Métodos}

Estudio observacional descriptivo, en pacientes hospitalizados con patología quirúrgica del servicio de cirugía general del Hospital de San José entre marzo de 2009 y agosto de 2009. Se incluyeron aquellos con hospitalización mayor de 24 horas que no tuvieron asistencia del grupo de soporte nutricional. Se excluyeron pacientes menores de 18 años, los de la unidad de cuidado intensivo, los programados para cirugía bariátrica, aquellos que presentaran enfermedades inflamatorias intestinales diagnosticadas por estudio histopatológico o por historia clínica y los pacientes con VIH por historia clínica. A los que cumplieron con los criterios de inclusión se les aplicó la escala global subjetiva entre las 24 y 72 horas de la hospitalización.

La escala global subjetiva incluye dos partes: la historia clínica y el examen físico. La primera está dirigida a conocer la evolución del peso del paciente en los seis meses anteriores y en los últimos quince días, cambios en la ingesta de nutrientes y su duración, alteraciones de la capacidad funcional y por cuánto tiempo, y grado de estrés de acuerdo con la enfermedad. El examen físico valora la pérdida de masa muscular y de grasa, así como la presencia de ascitis, edema maleolar y de región sacra. Al final de la evaluación el paciente se clasifica en una de las siguientes categorías según las cifras asignadas en la escala: bien nutrido (A), moderadamente desnutrido (B) y desnutrido grave (C) (Anexo 1).

Se midieron variables como diagnóstico de ingreso, estrato, estancia hospitalaria (número de días), comorbilidades (antecedentes patológicos valorados al momento del ingreso al estudio), condición quirúrgica (estado quirúrgico), el tipo de cirugía (abierta $v s$. laparoscópica), zona anatómica (área donde se realiza la cirugía) y presencia de infección en la herida quirúrgica. Se calcularon frecuencias absolutas, relativas, 
medidas de tendencia central y dispersión utilizando STATA 10. Este estudio se ajusta a las consideraciones del código de Nuremberg, declaración de Helsinki 2008 e informe Belmont y corresponde a un estudio sin riesgo sobre los pacientes, contemplado en el artículo 11 de la Resolución 8430 del Ministerio de la Protección Social de Colombia.

\section{Resultados}

Se incluyeron 281 pacientes hospitalizados del servicio de cirugía general, de los cuales $156(55,5 \%)$ eran hombres. La mediana de la edad fue 45 años y los días de estancia fue prolongada en los pacientes malnutridos (Tabla 1). El estrato socioeconómico tres predominó en los malnutridos. La prevalencia de malnutrición de la población fue $45,2 \%$, moderada $32,4 \%$ y severa el $12,8 \%$. De todos los malnutridos el $68 \%$ tuvo una estancia hospitalaria igual y/o superior a ocho días. La mayoría con edad igual o superior a 45 años. Los tres diagnósticos de ingreso más frecuentes fueron patología biliar, apendicitis aguda y pancreatitis aguda (Tabla 2).

\begin{tabular}{|c|c|c|c|}
\hline \multicolumn{4}{|c|}{$\begin{array}{c}\text { Tabla I. Características de la población de } \\
\text { estudio según estado nutricional }\end{array}$} \\
\hline Características n (\%) & $\begin{array}{l}\text { Bien } \\
\text { nutrido } \\
\mathrm{N}=154\end{array}$ & $\begin{array}{c}\text { Mal nutrido } \\
\text { moderado } \\
\mathrm{N}=91\end{array}$ & $\begin{array}{l}\text { Mal nutrido } \\
\text { severo } \\
\mathrm{N}=36\end{array}$ \\
\hline \multicolumn{4}{|l|}{ Sexo } \\
\hline$F$ & 74 (48) & $37(40.6)$ & $14(38,9)$ \\
\hline$M$ & $80(51.9)$ & $54(54)$ & $22(6 \mathrm{I})$ \\
\hline \multicolumn{4}{|l|}{$\begin{array}{l}\text { Estrato } \\
\text { socioeconómico }\end{array}$} \\
\hline I & $3(2)$ & $2(2)$ & 0 \\
\hline 2 & $40(26)$ & $30(33)$ & $22(6 \mathrm{I})$ \\
\hline 3 & 89 (58) & $52(57)$ & $13(36)$ \\
\hline 4 & $18(12)$ & $7(8)$ & I (3) \\
\hline 5 & $4(3)$ & 0 & 0 \\
\hline Edad mediana (RIQ)* & $38.5(28-50)$ & $54(42-65)$ & $60(48-72)$ \\
\hline Días mediana (RIQ)* & $4(3-5)$ & $8(7-10)$ & II (9-17) \\
\hline
\end{tabular}

* Rango intercuartílico

\begin{tabular}{|l|c|c|}
\hline \multicolumn{3}{|c|}{$\begin{array}{c}\text { Tabla 2. Edad, estancia y diagnóstico de ingreso } \\
\text { según estado nutricional }\end{array}$} \\
\hline Característica n (\%) & $\begin{array}{c}\text { Bien nutrido } \\
\text { N=I54 }\end{array}$ & $\begin{array}{c}\text { Mal nutrido } \\
\text { N=I27 }\end{array}$ \\
\hline Edad (años) & & \\
\hline Mayores de 45 & $50(32)$ & $90(71)$ \\
\hline Estancia (días) & & \\
\hline I-7 & $145(94)$ & $40(32)$ \\
\hline $8-14$ & $7(5)$ & $59(46)$ \\
\hline Mayor de 15 & $2(1)$ & $28(22)$ \\
\hline Diagnóstico de ingreso & & $16(13)$ \\
\hline Patología biliar & $40(26)$ & $10(8)$ \\
\hline Apendicitis aguda & $39(25)$ & $11(9)$ \\
\hline Pancreatitis aguda & $2(1)$ & $8(6)$ \\
\hline Enf. diverticular & $1(1)$ & $17(13)$ \\
\hline Traumáticas & $21(14)$ & $4(3)$ \\
\hline Enf. esofágicas & 0 & 0 \\
\hline Eventración & $9(6)$ & $14(11)$ \\
\hline Vasculares & $10(6)$ & $27(21)$ \\
\hline Tumor gastrointestinal & $1(1)$ & $20(16)$ \\
\hline Otros* & $31(20)$ & \\
\hline
\end{tabular}

* Empiema, cáncer de tiroides, obstrucción intestinal, úlcera duodenal, absceso glúteo, prolapso rectal, hernia inguinal, umbilical, púrpura trombocitopénica idiopática.

Al ingreso del estudio el $36 \%$ de los pacientes malnutridos tenían historia de enfermedades cardiovasculares. Sin embargo, es importante resaltar que la mayoría no presentaron ningún antecedente patológico (Tabla 3). De todos los pacientes con malnutrición, el $65 \%$ son posquirúrgicos. La zona anatómica más común encontrada con malnutrición fue la abdominal, las siguientes fueron la torácica $13 \%$ y la vascular con $12 \%$. Del grupo de los malnutridos la cirugía abierta tiene una mayor frecuencia que la laparoscópica (Tabla 4). En el mismo grupo el $89 \%$ no presentó infección del sitio operatorio (Tabla 5). 


\begin{tabular}{|c|c|c|}
\hline Comorbilidades n (\%) & $\begin{array}{c}\text { Bien nutrido } \\
n=154\end{array}$ & $\begin{array}{c}\text { Mal nutrido } \\
n=127\end{array}$ \\
\hline \multicolumn{3}{|l|}{ Cardiovasculares } \\
\hline No & |4| (92) & $81(64)$ \\
\hline Sí & $13(8)$ & $46(36)$ \\
\hline \multicolumn{3}{|l|}{ Pulmonar } \\
\hline No & $145(94)$ & $98(77)$ \\
\hline Sí & $9(6)$ & $29(23)$ \\
\hline \multicolumn{3}{|l|}{ Renal } \\
\hline No & $150(97)$ & $119(94)$ \\
\hline Sí & $4(3)$ & $8(6)$ \\
\hline \multicolumn{3}{|l|}{ Metabólico } \\
\hline No & $143(93)$ & $112(88)$ \\
\hline Sí & $11(7)$ & $15(12)$ \\
\hline \multicolumn{3}{|l|}{ Reumatológicas } \\
\hline No & $152(99)$ & $123(97)$ \\
\hline Sí & $2(1)$ & $4(3)$ \\
\hline \multicolumn{3}{|l|}{ Malignas } \\
\hline No & I5I (98) & $124(98)$ \\
\hline Sí & $3(2)$ & $3(2)$ \\
\hline \multicolumn{3}{|l|}{ Hepáticas } \\
\hline No & $153(99)$ & $127(100)$ \\
\hline Sí & I (I) & 0 \\
\hline Ninguna & $32(21)$ & $75(59)$ \\
\hline
\end{tabular}

$\begin{aligned} & \text { Tabla 4. Variables quirúrgicas según estado } \\
& \text { nutricional }\end{aligned}$
\begin{tabular}{l|c|c|}
\hline Característica n (\%) & $\begin{array}{c}\text { Bien nutrido } \\
n=154\end{array}$ & $\begin{array}{c}\text { Mal nutrido } \\
n=127\end{array}$ \\
\hline Condición quirúrgica & $27(18)$ & $45(35)$ \\
\hline Prequirúrgica & $127(82)$ & $82(65)$ \\
\hline Posquirúrgica & $11(7)$ & $17(13)$ \\
\hline Zona anatómica & $113(73)$ & $89(70)$ \\
\hline Tórax & $13(9)$ & $4(3)$ \\
\hline Abdomen & $11(7)$ & $15(12)$ \\
\hline Cabeza y cuello & $6(4)$ & $2(2)$ \\
\hline Vascular & $31(20)$ & $14(11)$ \\
\hline Otro & $96(62)$ & $69(54)$ \\
\hline Tipo de cirugía & $27(18)$ & $44(35)$ \\
\hline Laparoscopia & & \\
\hline Abierta & & \\
\hline Ninguno & & \\
\hline
\end{tabular}

\begin{tabular}{|l|c|c|}
\hline \multicolumn{3}{|c|}{ Tabla 5. Infección del sitio operatorio según } \\
estado nutricional \\
\hline Característica n (\%) & Bien nutrido & Desnutrido \\
\hline ISO & & \\
\hline Sí & $15 / 154(10)$ & $14 / 127(11)$ \\
\hline No & $139 / 154(90)$ & $1 / 3 / 127(89)$ \\
\hline ISO inicial & & \\
\hline Superficial & $12 / 15(80)$ & $6 / 14(43)$ \\
\hline Profunda & $3 / 15(20)$ & $6 / 14(43)$ \\
\hline Órgano y/o espacio & $0 / 15(0)$ & $2 / 14(14)$ \\
\hline ISO definitiva & & \\
\hline Superficial & $11 / 15(73)$ & $6 / 14(43)$ \\
\hline Profunda & $4 / 15(27)$ & $6 / 14(43)$ \\
\hline Órgano y/o espacio & $0 / 15(0)$ & $2 / 14(14)$ \\
\hline
\end{tabular}

\section{Discusión}

La malnutrición es un trastorno metabólico causado por el desequilibrio entre la ingesta de nutrientes y las necesidades corporales. Tiene prevalencia en países en vía de desarrollo por la carencia de alimentos en cantidad y calidad suficientes. En el paciente hospitalizado fue descrita desde hace más de 30 años por el Dr. Butterwotrh ${ }^{8}$ cuando publicó varios casos que dado su estado nutricional no tenían mejoría. En Norteamérica la prevalencia reportada es del $40 \%$, mientras que en Latinoamérica la $\mathrm{MH}$ es de hasta el $50 \%$ con importantes implicaciones médicas en su recuperación, cicatrización de los tejidos y estancias hospitalarias prolongadas. ${ }^{2} \mathrm{Se}$ ha intentado con estos estudios de prevalencia de malnutrición y otros donde muestran los problemas relacionados con ellas ${ }^{4,5,9,10}$ crear conciencia en los profesionales de la salud en el registro y tratamiento de la malnutrición; pero hasta ahora no se ha logrado obtener un adecuado registro de la valoración nutricional en la historia clínica por parte del cuerpo médico, tal como se anotan, por ejemplo, los signos vitales.

En nuestro hospital a los pacientes que ingresan no se les realiza un registro nutricional a pesar de que las cifras de malnutrición hospitalaria en Latinoamérica son cercanas al 50\%; siendo entonces un gran mal subestimado por todos los profesionales de la salud y que tiene un impacto directo en la evolución médica de nuestros pacientes. ${ }^{2}$ En 
el Hospital de San José, servicio de cirugía general, se realizó el primer estudio de malnutrición hospitalaria en conjunto con el servicio de nutrición clínica. Allí ingresaron pacientes provenientes de urgencias y consulta externa, a quienes se implementó la VSG instrumento sencillo y ágil ${ }^{3}$ aplicado por el médico para evaluar el estado nutricional y así poder tomar decisiones terapéuticas. Históricamente se ha estudiado la valoración global subjetiva la cual se ha avalado en múltiples estudios, en especial el de Detsky 1987 que utiliza este instrumento y se correlaciona con los niveles séricos de albúmina. ${ }^{3}$

En nuestro estudio se analizaron 281 pacientes durante seis meses, con una prevalencia de malnutrición del $45,2 \%$, que es alta, pero se encuentra cerca de la reportada en la literatura médica latinoamericana. El estudio IBRANUTRI, análisis multicéntrico con 4.000 pacientes hospitalizados informó una prevalencia de 48,1\% de malnutrición. ${ }^{2}$ Sin embargo, consideramos que a pesar que los resultados encontrados en nuestro estudio coinciden con lo reportado en Latinoamérica, sigue siendo una cifra alarmante y es necesario actuar y detectar la malnutrición en nuestros pacientes y así disminuir no sólo este porcentaje sino también las posibles complicaciones en los hospitalizados que es casi de $20 \%$ en Latinoamérica. ${ }^{6}$

El estrato socioeconómico tres predominó en este estudio, que es el de la mayoría de la población general que atiende el hospital, lo cual no permite considerar que haya relación entre el estado nutricional hospitalario y este tipo socioeconómico, concordante con la literatura médica americana. El estudio IBANUTRI sugiere que el estrato socioeconómico bajo puede ser un factor de riesgo para $\mathrm{MH}{ }^{1}$

La literatura latinoamericana señala que la media de estancia hospitalaria para los pacientes malnutridos es de cerca de nueve días, lo cual es concordante con los resultados de esta investigación. Nuestros pacientes malnutridos presentaron una estancia mayor o igual a ocho días. La literatura reporta que hay asociación entre malnutrición y aumento en la estancia hospitalaria, a esto se le suman factores médicos que influyen como las comorbilidades cardiovasculares que aumentan los costos hospitalarios hasta un $300 \% .{ }^{3}$ En nuestro estudio no es posible establecer una medida de asociación entre malnutrición y comorbilidades, ya que la mayoría de nuestra población evaluada es joven y no las presentó.

Para este estudio se debe considerar que la estancia hospitalaria de nuestros pacientes fue tomada de las historias clínicas después de la valoración de su estado nutricional por medio de la escala global subjetiva. En la literatura médica se reporta un aumento en el costo hasta un $60 \%$, asociado sólo con $\mathrm{MH}^{3}$ y si bien es un tema importante, nuestro estudio no tenía como objeto analizar esta relación y sería un gran capítulo para investigar y determinar el costo-efectividad en nuestros casos. La mayor población desnutrida está por encima de 45 años y esto lo relaciona de forma muy especial la literatura latinoamericana con mayor probabilidad de malnutrición ${ }^{1,6,11}$ exacerbado además por la enfermedad de base que los hizo consultar.

El diagnóstico de ingreso y la zona anatómica más frecuente encontrada en este estudio fue la esperada, ya que está relacionada con las causas más frecuentes de consulta del hospital. Sin embargo, es importante analizar los procedimientos en tórax y vasculatura por su alta malnutrición. Consideramos que estas zonas anatómicas están expuestas a procedimientos de alta complejidad que deben explorarse en futuros estudios. La mayoría de los pacientes malnutridos son los posquirúrgicos, explicado en la literatura científica porque su catabolismo se aumenta y al no corregir las demandas con el aporte adecuado, dan lugar al gran capítulo de malnutrición hospitalaria. Además en este estudio no se valoró la mortalidad, lo cual es ampliamente discutido en la literatura latinoamericana ${ }^{1,3,5}$ al revelar una relación entre mortalidad y malnutrición. La infección del sitio operatorio se presentó en el $11 \%$ de la población malnutrida, pero resulta difícil compararlo pues no hay informes claros en la literatura sobre asociación de malnutrición e infección quirúrgica. El estudio realizado por Schneider intentó determinar la relación entre malnutrición hospitalaria e infección nosocomial; menciona que el tamizaje nutricional temprano disminuiría el riesgo de presentar esta última. ${ }^{5}$

Concluimos que nuestra prevalencia de $\mathrm{MH}$ es alta por lo cual debemos en nuestro hospital aplicar la VGS a todos los pacientes hospitalizados y así podermos clasificar y actuar de manera precoz con el grupo de soporte nutricional para evitar las múltiples consecuencias que trae la malnutrición hospitalaria. 


\section{Anexo 1. Valoración global subjetiva}

Paciente:

edad:

talla:

Servicio:

cama:

S.S.:

Diagnóstico:

\section{A. HISTORIA}

1. Cambios en el peso corporal

Pérdida total de peso en los últimos seis meses

Cambios las últimas 2 semanas:

$\mathrm{kg} \%$ de pérdida.

aumento

ninguno

disminución

2. Cambios en la dieta en relación con lo normal (antes de la hospitalización)

Sin cambios:

Cambio:

duración:

semanas:

Tipo:__ dieta sólida insuficiente:

dieta líquida hipocalórica: dieta líquida total:

ayuno:

3. Síntomas gastrointestinales (persistentes por más de dos semanas)

Ninguno: náusea: vómito: diarrea: anorexia:

\section{Capacidad funcional}

No hay disfunción

Disfunción: duración: semanas:

Tipo: disminución en trabajo: ambulatorio:

reducido lecho:

B. EXAMEN FÍSICO (en cada punto, calificar normal $=0$, leve $=1$, moderado $=2$, severo $=3$ )

Pérdida de grasa subcutánea (tríceps, tórax)

Atrofia muscular (cuádriceps, deltoides)

Edema de tobillo edema sacro ascitis

\section{CALIFICACIÓN DE LAVALORACIÓN GLOBALSUBJETIVA}

Bien nutrido:

Moderadamente desnutrido o con riesgo de desnutrición

Severamente desnutrido
A:

B:

C:

\section{Referencias}

1. Baccaro F, Moreno JB, Borlenghi C, Aquino L, Armesto G, Plaza G, et al. Subjective global assessment in the clinical setting. JPEN J Parenter Enteral Nutr. 2007 Sep;31(5):406-9.

2. Waitzberg DL, Caiaffa WT, Correia MI. Hospital malnutrition: the Brazilian national survey (IBRANUTRI): a study of 4000 patients. Nutrition 2001 Jul; 17(7-8):573-80

3. Detsky AS, McLaughlin JR, Baker JP, Johnston N, Whittaker S, Mendelson $\mathrm{RA}$, et al. What is subjective global assessment of nutritional status? JPEN J Parenter Enteral Nutr 1987 Jan;11(1):8-13

4. Correia MI, Campos AC. Prevalence of hospital malnutrition in Latin America: the multicenter ELAN study. Nutrition. 2003 Oct;19(10):823-5

5. Pablo AM, Izaga MA, Alday LA. Assessment of nutritional status on hospital admission: nutritional scores. Eur J Clin Nutr 2003 Jul;57(7):824-31
6. Correia MI, Waitzberg DL. The impact of malnutrition on morbidity, mortality, length of hospital stay and costs evaluated through a multivariate model analysis. Clin Nutr. 2003 Jun;22(3):235-9

7. Elia M, Zellipour L, Stratton RJ. To screen or not to screen for adult malnutrition? Clin Nutr. 2005 Dec;24(6):867-84.

8. Butterworth CE, Jr. [The skeleton in the hospital closet. 1974]. Nutr Hosp 2005 Jul;20(4):302-7, 301

9. Mullen JL. Consequences of malnutrition in the surgical patient. Surg Clin North Am 1981 Jun;61(3):465-87.

10. Weinsier RL, Hunker EM, Krumdieck CL, Butterworth CE. Hospital malnutrition. A prospective evaluation of general medical patients during the course of hospitalization. Am J Clin Nutr. 1979 Feb; 32(2):418-26.

11. Sacks GS, Dearman K, Replogle WH, Cora VL, Meeks M, Canada T. Use of subjective global assessment to identify nutrition-associated complications and death in geriatric long-term care facility residents. J Am Coll Nutr. 2000 Oct;19(5):570-7. 